\title{
Fischer, J. (2020). Willkommen im 21. Jahrhundert. Europas Aufbruch und die deutsche Verantwortung
}

Köln: Kiepenheuer \& Witsch. 208 S., ISBN: 978-3-462-05473-6, $€ 20,-$.

\section{Sven Morgen}

Online publiziert: 22. Oktober 2020

(C) Der/die Autor(en) 2020

Manchmal dauern Jahrhunderte länger als genau 100 Jahre und manchmal beginnt deswegen ein neues Jahrhundert etwas später. So zum Beispiel das 21. Jahrhundert. Wir schreiben zwar schon das Jahr 2020, aber so richtig werden die Konturen des 21. Jahrhunderts erst jetzt erkennbar. Nach dem fälschlicherweise angenommenen Ende der Geschichte in den 1990ern und einem Jahrzehnt der amerikanischen Superhegemonie am Anfang der 2000er, die mit dem Krieg gegen den Terror ihr eigenes Ende geschaffen hat, können wir aktuell den Beginn einer Zeitenwende und globalen Machtverschiebung beobachten. Joschka Fischer bietet in seinem Buch Willkommen im 21. Jahrhundert. Europas Aufbruch und die deutsche Verantwortung aus deutscher und europäischer Perspektive einen interessanten Blick auf die bereits stattfindenden und auf die noch bevorstehenden, aber bereits schemenhaft erkennbaren, geopolitischen Entwicklungen und Umbrüche an.

Fischer startet sein Buch mit der Feststellung, dass aktuell drei Revolutionen gleichzeitig bevorstehen, die die Welt grundlegend verändern werden und Deutschland und Europa vor größte Herausforderung stellen. Als erstes wird die Revolution des transatlantischen Verhältnisses durch die Präsidentschaft Donald Trumps angeführt. Diese bedingt, dass Europa zukünftig nicht mehr vorbehaltslos auf die (sicherheitspolitische) Unterstützung der USA zählen könne. Als zweite Revolution müsse der Aufstieg Chinas zur globalen Nummer eins gesehen werden, der die geopolitischen und wirtschaftlichen Machtverhältnisse nachhaltig neu ordnen und Konsequenzen für Europas Selbstverständnis sowie das transatlantische Verhältnis haben wird. Als dritten Punkt führt Fischer die Digitale Revolution an, von der er prognostiziert, dass diese ebenso geeignet ist, die globalen Machtverhältnisse

S. Morgen ( $\square)$

Lehrstuhl für Internationale Beziehungen, Friedrich-Schiller-Universität Jena,

Carl-Zeiß-Straße 3, 07743 Jena, Deutschland

E-Mail: sven.morgen@uni-jena.de 
vollkommen neu zu ordnen und Fragen nach Souveränität und Abhängigkeit im 21. Jahrhundert virulent zu machen. Fischer geht davon aus, dass es mit diesen Revolutionen auch eine längere Phase der globalen Unordnung und des erhöhten Risikos geben wird und spitzt diese Gemengelage auf folgende Frage zu: „Wird Europa also im 21. Jahrhundert ein Leben unter fremder Einflussnahme und in wirtschaftlicher Abhängigkeit zu führen haben?“'(S. 27). Die Beantwortung dieser Frage hängt für Fischer allein vom Willen der Europäer zur eigenen Souveränität ab.

Wie Europa und Deutschland diese Souveränität erlangen und auch zukünftig erhalten könnte, führt Fischer in den einzelnen Kapiteln seines Buches aus. Zunächst müssten Europa und Deutschland anerkennen, dass der von Trump erzwungene Wandel im transatlantischen Verhältnis nachhaltig andauern wird - auch unabhängig von der Person Trump und dessen Nachfolger. Europa müsse die erzwungene „gaullistische Wende“ (S. 57) anerkennen und eine eigene geostrategische Identität ausbilden, eigene Interessen formulieren und die zur Verteidigung dieser Interessen notwendigen politischen Mittel entwickeln. Damit Europa diesen Schritt vollziehen kann, müsse jedoch zunächst die deutsche Frage beantwortet werden. Nämlich die Frage, wie und ob Deutschland sein Verhältnis zu den ihm innewohnenden Machtpotenzial angehen will. Nach Fischer wird ein gaullistischer Aufbruch Europas nur möglich sein, wenn Deutschland eine Änderung seines Selbstverständnisses erzwingt und ein positives Verhältnis zu seiner Macht entwickelt. Der Autor plädiert dafür, dass Deutschland ,seine tatsächlich vorhandene große zivile Macht, seine finanzielle Stärke“ (S. 91) für ein souveränes Europa einsetzen soll.

Diese Souveränität sei unter anderem durch die digitale Revolution gefährdet. Nämlich dann, wenn Europa den Anschluss an die Weltspitze verliert und entweder von den USA oder China abhängig wird. Die Digitalisierung und Entwicklungen in der Künstlichen Intelligenz haben das Potenzial, nicht nur eine technische, sondern auch eine ökonomische und gesellschaftliche Revolution auszulösen, die weitreichende Folgen für die Verteilung der globalen Vermögenswerte und Wirtschaftspotenziale hat. Unternehmen und Volkswirtschaften, die auf diese digitale Revolution nicht reagieren oder den Anschluss verlieren, geraten ins Hintertreffen und werden „,durch Souveränitätsverlust, Abhängigkeit, Fremdbestimmung und Wohlstandsverlust bestraft"“ (S. 107).

Die dritte große Revolution ist der Aufstiegs Chinas und die damit aufkommende Systemkonfrontation, in der sich Chinas als Systemalternative zum Westen darstellt und die Zukunft der westlich-liberalen Demokratie gefährdet. Für Fischer stellt der ,systematische Angriff auf die individuelle Freiheit, auf das Fundament der westlichen Demokratie“ (S. 176) die größte Herausforderung durch China dar. Der eigentliche Systemwiderspruch im 21. Jahrhundert bestünde im Widerspruch zwischen einer westlichen Freiheitskultur und einer chinesischen konfuzianisch geprägten, mit repressiven Mitteln erzwungenen Harmoniekultur. Dabei werde das westliche System zum ersten Mal von einem Gegner herausgefordert, der sich mit seinem höchst erfolgreichen totalitären Ansatz zu einer attraktiven Alternative der kapitalistischen Moderne entwickelt.

Fischer fasst seine Ausführungen konsequent zusammen, indem er feststellt, dass das strategische Ziel der EU sein muss, sich technologisch in der globalen Spitzengruppe zu halten und machtpolitisch so stark zu werden, dass es sich selbst 
verteidigen kann. „Für Europa zählen keine Statusfragen, sondern Souveränitätsfragen, Status setzt Souveränität voraus“ (S. 192). Und genau diese Souveränität drohen Deutschland und Europa zu verlieren, wenn sie nicht adäquat auf die drei großen Revolutionen reagieren - dies wird mit der Lektüre von Fischers Buch deutlich.

Eine vierte - in ihren potenziellen Auswirkungen noch viel größere - Revolution wird von Fischer zwar an einigen Stellen im Buch angesprochen, aber nicht ausreichend behandelt: der Klimawandel. Die verheerenden Auswirkungen eines ungebremsten Klimawandels haben das Potenzial sowohl die geopolitischen Verhältnisse nachhaltig zu erschüttern als auch die digitale Revolution zu stoppen. Will man das 21. Jahrhundert vorausdenken, müsste man eigentlich mit dem Kampf gegen den Klimawandel beginnen, da Erfolg oder Misserfolg in diesem Bereich die von Fischer klug skizzierten anderen Revolutionen maßgeblich beeinflussen werden.

Funding Open Access funding enabled and organized by Projekt DEAL.

Open Access Dieser Artikel wird unter der Creative Commons Namensnennung 4.0 International Lizenz veröffentlicht, welche die Nutzung, Vervielfältigung, Bearbeitung, Verbreitung und Wiedergabe in jeglichem Medium und Format erlaubt, sofern Sie den/die ursprünglichen Autor(en) und die Quelle ordnungsgemäß nennen, einen Link zur Creative Commons Lizenz beifügen und angeben, ob Änderungen vorgenommen wurden.

Die in diesem Artikel enthaltenen Bilder und sonstiges Drittmaterial unterliegen ebenfalls der genannten Creative Commons Lizenz, sofern sich aus der Abbildungslegende nichts anderes ergibt. Sofern das betreffende Material nicht unter der genannten Creative Commons Lizenz steht und die betreffende Handlung nicht nach gesetzlichen Vorschriften erlaubt ist, ist für die oben aufgeführten Weiterverwendungen des Materials die Einwilligung des jeweiligen Rechteinhabers einzuholen.

Weitere Details zur Lizenz entnehmen Sie bitte der Lizenzinformation auf http://creativecommons.org/ licenses/by/4.0/deed.de. 